NOTICE: this is the author's version of a work that was accepted for publication in Carbon. Changes resulting from the publishing process, such as peer review, editing, corrections, structural formatting, and other quality control mechanisms may not be reflected in this document. Changes may have been made to this work since it was submitted for publication. A definitive version was subsequently published in Carbon, Vol. 63 (2013). DOI:

10.1016/j.carbon.2013.07.002 


\title{
An Energy Landscape for Carbon Network Solids
}

\author{
R.C. Powles ${ }^{1}$, N.A. Marks ${ }^{2 *}$, D.W.M. Lau ${ }^{3}$, D.G. McCulloch ${ }^{4}$ and D.R. McKenzie ${ }^{1}$ \\ ${ }^{1}$ School of Physics, The University of Sydney, Sydney NSW 2006, Australia \\ ${ }^{2}$ Nanochemistry Research Institute, Curtin University, GPO Box U1987, Perth WA 6845, Australia \\ ${ }^{3}$ School of Physics, The University of Melbourne, Melbourne VIC 3010, Australia \\ ${ }^{4}$ School of Applied Sciences, RMIT University, GPO Box 2476V, Melbourne VIC 3001, Australia
}

\begin{abstract}
Carbon network solids show a rich diversity with many distinct structural classes. Transitions between classes can be induced by annealing and mechanical compression, frequently with unexpected results. We have constructed an energy landscape based on atomistic simulations that includes both amorphous and crystalline bonded networks. The landscape, representing a minimum free energy surface, is constructed as a function of density and the degree of crystallinity and is used to explain experimental observations. We use the landscape to explain 1) why some carbon structures show full recovery from deformation while others deform permanently 2) why annealing of non-crystalline materials grown in a low pressure pure carbon vapor quenching process always lead ultimately to graphite and not to diamond and 3) why room temperature compression of graphitic carbons leads to a reversible amorphization. The tetrahedrally bonded amorphous structure is predicted to have the lowest free energy at sufficiently high pressures and temperatures and therefore is expected to occur as the endpoint of rapidly quenched shock compression processes of carbon structures.
\end{abstract}

*Corresponding author. E-mail address: N.Marks@curtin.edu.au (N.A. Marks) 


\section{Introduction}

All known crystalline structures of elemental carbon, including graphite, diamond and their polytypes contain only one hybridization state of the carbon atom (either $s p^{2}$ or $s p^{3}$ ). Non-crystalline carbon, in contrast, can contain $s p, s p^{2}$ and $s p^{3}$ hybridizations as well as mixed states. The ability of $s p^{2}$ atoms to form chain-like, ribbon-like or sheet-like structures introduces topological complexity leading to many non-crystalline forms of carbon in structurally distinct classes $[1,2]$. For example, graphene sheets containing non-six-membered rings, given freedom to fill three-dimensional space, acquire positive or negative curvature, align in many ways and can form voids [3]. In some structures, $\mathrm{sp}^{3}$ crosslinks between graphene sheets increase the rigidity [4]. Well known three dimensionally disordered structures are the glassy carbons produced by pyrolysis [5], low density amorphous carbons with a dominance of $s p^{2}$ hybridized carbon (frequently termed as "amorphous carbon" or $a-\mathrm{C}[6])$ and high density, $a-\mathrm{C}$ which contain a dominance of $s p^{3}$ hybridized carbon (termed as "tetrahedral amorphous carbon" or $t a-\mathrm{C})$ [7].

Although the conventional synthetic route from graphite to diamond driven by high temperatures and pressures is well understood [8], the rules that govern transformations between crystalline and non-crystalline carbon and between the various non-crystalline classes of carbon are unknown despite the large amount of observational data. For example, the results of compression of crystalline and non-crystalline carbon at room temperature are diverse and sometimes surprising. Using diamond anvils it has been found [9-13] that there is a reversible transformation at room temperature between the $s p^{2}$ hybridized starting material to a majority $s p^{3}$ hybridized state at pressures above approximately $15 \mathrm{GPa}$. The nature of these high pressure structures and the reasons for an apparently reversible amorphization process induced by pressure alone are unresolved [12]. Some carbon materials show elastic behavior during deformation [14], while others show inelastic behavior with a remarkable ability to recover shape completely after deformation [15]. There are also unresolved phenomena surrounding transformations between non-crystalline carbons at elevated temperatures. For example, after a thermal anneal, some forms of $a$-C reduce their $s p^{3}$ content [16] while 
others maintain or even increase their $s p^{3}$ content [17-19]. Recently, extremely rapid non equilibrium crystallization of $a$-C has been demonstrated using x-ray pulses [20]. An x-ray pulse delivers energy to the electrons, causing bonding changes under non equilibrium conditions. An understanding of the diverse behavior of crystalline and non-crystalline carbon as a function of pressure and temperature is central to important application areas of carbon films, including low friction and wear resistant coatings for high performance applications [21] and emerging applications in fast optical memories [22]

In this paper, we provide an understanding of the diverse behavior of carbon network solids by the construction of an energy landscape which enables visualization of the pathways between various carbon structures. The shape of the landscape is a conceptual rendition of the results of a series of molecular dynamics (MD) simulations which calculate the energy per atom of key synthesized structures. The landscape is used to understand and unify diverse observations from experiments by interpreting them as movements on an appropriate energy landscape. 


\section{Computational Methods}

The MD simulations utilize the environment-dependent interaction potential (EDIP) [23] which provides a realistic and computationally efficient description of carbon bonding. In EDIP, the interaction Hamiltonian is a sum over atom sites with terms corresponding to two- and three- body interactions. This potential has been benchmarked against ab initio calculations [24] and deals well with the diversity of structure in carbon including intermediate hybridization states which are important for the description of locally strained configurations containing minority bond types. EDIP has been successfully used to describe a variety of phenomena including $a-\mathrm{C}$ thin-film growth [25], carbon onion formation [26] and creation of nanodiamond [27].

The energy of systems containing large numbers of atoms with independent positional coordinates can be represented by a surface in a space of high dimension $(3 N+1$ where $N$ is the number of atoms). This high dimensionality prevents convenient visualization of the energy landscape of the system. A solution to this problem is to project the landscape onto a low dimensional space by calculating a small number of structural parameters. The resulting energy is not a unique function of these structural parameters but an appropriate choice of them minimizes the spread of energies at neighboring values of the structural parameters. In this paper, we describe the landscape in terms of the Gibbs free energy per atom, $g$, defined in terms of pressure $(P)$ and temperature $(T)$ by

$$
\mathrm{g}=\mathrm{u}+\mathrm{Pv}-\mathrm{Ts}
$$

where $u$ is the potential energy, $v$ is the volume and $s$ is the entropy per atom. Structural parameters of density $(\rho)$ and crystalline order $(\chi)$ are chosen as they are directly related to specific $v$ and entropy $s$ 
respectively. This choice enables the evaluation of $g$ at different temperatures and pressures. The crystalline order parameter $\chi$ is defined by,

$$
\chi=\frac{N_{3}}{N}\left(1-\frac{\sigma_{3}}{\sigma_{0}}\right)+\frac{N_{4}}{N}\left(1-\frac{\sigma_{4}}{\sigma_{0}}\right)
$$

where $N_{3}$ is the number of $\mathrm{sp}^{2}$ bonded atoms with only $\mathrm{sp}^{2}$ bonded neighbors; $\mathrm{N}_{4}$ is the corresponding quantity for $\mathrm{sp}^{3}$ atoms. This measure identifies atoms contained in clusters of similarly bonded atoms. The quantities $\sigma_{3}$ and $\sigma_{4}$ are the full width at half maximum for the first nearest-neighbour peak in the partial radial distribution functions for three and four-fold coordinated atoms respectively; $\sigma_{0}$ corresponds to a maximally disordered structure and was taken as 0.3 throughout. $\chi$ has the value of zero for a fully amorphous structure and unity for a perfect crystal and is designed to apply to all carbon structures, regardless of the ratio of the $\mathrm{sp}^{2}$ to $\mathrm{sp}^{3}$ atoms.

Carbon liquids containing 2000 atoms were generated at a range of densities (from 1.5 to $3.5 \mathrm{~g} / \mathrm{cm}^{3}$ ) in simulation cells with a side length ratio of 1:1:2 to facilitate later unixial compression. The liquids were prepared at $5000 \mathrm{~K}$ using a distorted simple cubic lattice which spontaneously melts [24]. After equilibration, the liquids were cooled to $300 \mathrm{~K}$ in $0.5 \mathrm{ps}$ to give structures with a high degree of disorder. Following previous work on nanotubes [28], onions [26] and peapods [29], these amorphous structures were subsequently annealed at $3500 \mathrm{~K}$ to produce structures with increasing order. This temperature is below the melting point of carbon which under EDIP is approximately $4500 \mathrm{~K}$, in close agreement with observations and calculations on graphite and diamond [30]. The evolution of the structure was followed for times between 0.1 and 0.9 ns. At intervals of 5 ps during each anneal, a copy was made of the instantaneous state of the system for analysis purposes. This copy was quenched to zero temperature by steepest descent minimization and then the structure was relaxed to its minimum potential energy by isotropically rescaling the coordinates and cell parameters. A series of rescaled structures were employed to search for the lowest 
potential energy, and after each rescaling the zero-temperature condition was again applied. The result is a structure that mirrors the relative atom positions at a temperature of absolute zero at the natural density of the structure when no pressure is applied.

To investigate the response of carbon structures to mechanical deformation, compression and decompression processes for four carbon structures were undertaken by repeatedly rescaling the system uniaxially along the long axis of the simulation cell. First the simulation cell was rescaled by $1 \%$ in twenty steps and the system evolved for 5 ps at $300 \mathrm{~K}$ at each step. These compressions increased the density by a total of $22 \%$. The subsequent decompression was carried out using the same number of steps, thereby returning the structure to its original density. At the conclusion of each compression and decompression cycle an analysis simulation was performed using the same zero temperature and pressure approach employed for the high-temperature annealing calculations.

\section{Results and Discussion}

Table 1 shows the densities of the structures (labeled $A$ through $L$ ) prepared by quenching liquids to $300 \mathrm{~K}$. Also shown are the densities of these structures following relaxation to zero pressure and temperature. Note that the highest density structures show a compressive stress after quenching since their density decreases substantially when they are relaxed at zero pressure. High compressive stresses of the order of $10 \mathrm{GPa}$ are usually observed when $t a-C$ is synthesized. In contrast, low density structures exhibit an increase in density when relaxed, consistent with the presence of tensile stress. 
Table 1 - A summary of the structures of Fig 1 including the density of the sample during the liquid quench from $5000 \mathrm{~K}$ to $300 \mathrm{~K}$ and the density before annealing and after annealing with the structure relaxed at zero pressure and temperature. Also shown are the $\mathrm{sp}^{2}$ and $\mathrm{sp}^{3}$ fractions, with the remaining atoms being sp bonded. The coordination was determined by counting neighbors within a cutoff of 0.185 nm.

\begin{tabular}{|c|c|c|c|c|c|c|c|}
\hline \multirow[t]{2}{*}{ Structure } & $\begin{array}{l}\text { Initial } \\
\text { Density }\end{array}$ & \multicolumn{3}{|c|}{ Before Annealing } & \multicolumn{3}{|c|}{$\begin{array}{l}\text { After annealing } \\
\text { for } 100 \mathrm{ps}\end{array}$} \\
\hline & & $\begin{array}{l}\text { Density } \\
\left(\mathrm{g} / \mathrm{cm}^{3}\right)\end{array}$ & $\begin{array}{c}\mathrm{sp}^{2} \\
\text { fraction }\end{array}$ & $\begin{array}{c}\mathrm{sp}^{3} \\
\text { fraction }\end{array}$ & $\begin{array}{l}\text { Density } \\
\left(\mathrm{g} / \mathrm{cm}^{3}\right)\end{array}$ & $\begin{array}{c}\mathrm{sp}^{2} \\
\text { fraction }\end{array}$ & $\begin{array}{c}\mathrm{sp}^{3} \\
\text { fraction }\end{array}$ \\
\hline$A$ & 1.5 & 1.548 & 55.4 & 6.1 & 1.543 & 87.4 & 10.5 \\
\hline$B$ & 2.0 & 2.115 & 64.8 & 16.8 & 2.062 & 80.5 & 16.6 \\
\hline$C$ & 2.3 & 2.438 & 63.4 & 26.0 & 2.331 & 83.6 & 15.1 \\
\hline$D$ & 2.4 & 2.547 & 60.9 & 31.9 & 2.483 & 70.3 & 28.3 \\
\hline$E$ & 2.5 & 2.626 & 61.0 & 33.2 & 2.59 & 71.5 & 27.2 \\
\hline$F$ & 2.6 & 2.717 & 57.0 & 39.3 & 2.692 & 57.6 & 40.7 \\
\hline$G$ & 2.75 & 2.856 & 52.6 & 45.3 & 2.863 & 49.9 & 49.5 \\
\hline$H$ & 2.9 & 2.973 & 48.2 & 50.9 & 2.983 & 44.9 & 54.8 \\
\hline$I$ & 3.0 & 3.032 & 45.1 & 54.2 & 3.078 & 40.4 & 59.5 \\
\hline$J$ & 3.1 & 3.07 & 44.1 & 55.5 & 3.123 & 36.7 & 63.1 \\
\hline$K$ & 3.25 & 3.129 & 41.7 & 58.0 & 3.204 & 31.0 & 69.0 \\
\hline$L$ & 3.5 & 3.206 & 38.7 & 61.2 & 3.25 & 28.7 & 71.2 \\
\hline
\end{tabular}


Fig. 1(a) shows the progression during annealing lasting 100 ps of $u(u=g$ for $\mathrm{P}=0$ and $\mathrm{T}=0)$ and average coordination (defined as the number of atoms within a radius of $0.185 \mathrm{~nm}$ ) for structures, labeled $A$ through $L$, summarized in Table 1. The spacing between points during annealing is a measure of the speed of evolution of the structure. The structures are evolving towards structures with a predominance of either 3fold or 4-fold coordinated atoms. Crystalline graphite and diamond are inferred to be the minimum energy structures that represent the end points of the trajectories. Fig. 2 shows example structures corresponding to structures $B$, and $\mathrm{I}$ in Fig. 1. Structure $I$ is highly disordered with a predominance of $\mathrm{sp}^{3}$ bonding and a homogenous (void free) microstructure, while structure B is mainly $\mathrm{sp}^{2}$ bonded with a high void fraction. Also shown in Fig. 2 are two annealed structures: structure $B$ ', obtained from 200 ps of annealing of structure $\mathrm{B}$, is largely $\mathrm{sp}^{2}$ bonded and contains graphene sheets that enclose voids. 

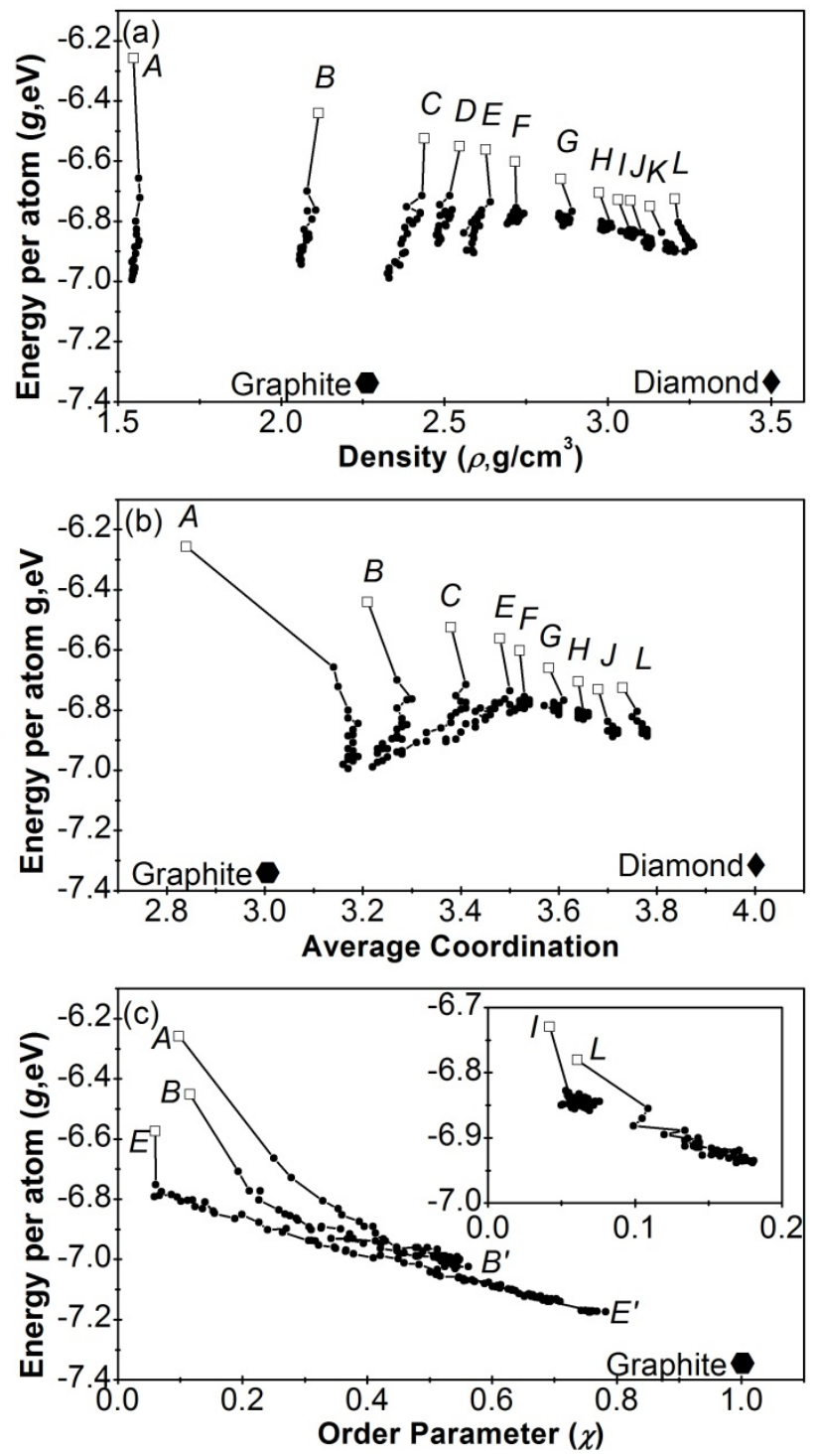

Fig. 1 - The progression of the free energy per atom $(g)$ during annealing at $3500 \mathrm{~K}$ in 5 ps time steps along with the structural parameters (a) average coordination, (b) density $(\rho)$ and (c) order parameter $(\chi)$ of equation (2). The locations of diamond and graphite are shown for reference. The labels $A-L$ refer to simulations of structures with increasing initial density. Panels (a) and (b) present data for a total annealing time of 100 ps. Data for longer annealing times (see text for details) is presented in panel (c). $B^{\prime}$ refers to the end point of structure $B$ after 200 ps of annealing, while $E^{\prime}$ is the endpoint of structure $\mathrm{E}$ after 900 ps of annealing. 
(a) Structure $B$

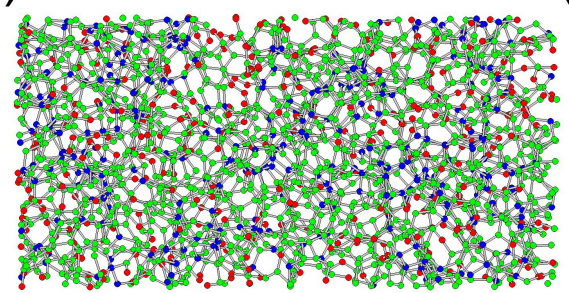

(c) Structure $E^{\prime}$

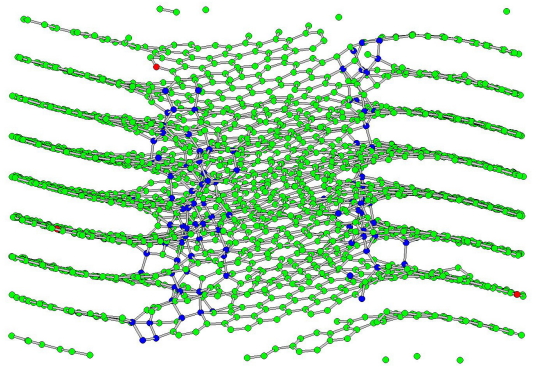

(b) Structure $B^{\prime}$

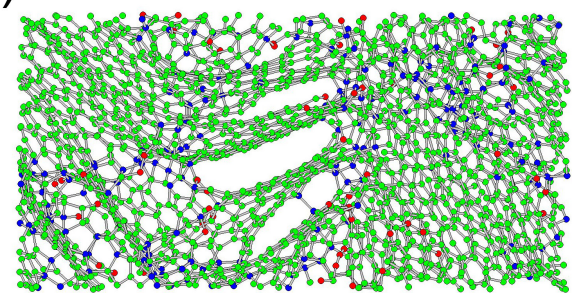

(d) Structure I

Fig. 2 - The structures at points (a) $B$, (b) $B^{\prime}$, (c) $E^{\prime}$ and d) $I$ from Fig. 1 with the color of the atoms indicating the coordination (2-fold: red, 3-fold: green and 4-fold: blue). Panels (a, b, and d) are sideviews oriented along a cartesian axis. The structure in panel (c) has been rotated to highlight the graphite-like order.

Fig. 1(b) shows the progression of $u$ and $\rho$ for selected structures from Fig. 1(a). Again, the data shown is for an annealing time period of 100 ps. The energy and structural parameters change quickly in the initial stages of the annealing and then their rate of change decreases. Fig. 1(c) shows the evolution of $u$ and $\chi$ for selected structures and reveals how the extent of the progression towards diamond and graphite depends on the density of the starting structure, with the lower density structures progressing to much higher levels of order for the same annealing time. In order to illustrate this progression clearly, structures A, B, I and L were annealed for a total time of 200 ps, while structure E was annealed for 900 ps (structure E'). After 200 ps the $\mathrm{sp}^{2}$ fraction in structures $\mathrm{A}, \mathrm{B}, \mathrm{E}$, I and L was $89.1 \%, 86.6 \%, 14.6 \%, 41 \%$ and $27.2 \%$, respectively. Only at the lower densities is much change seen relative to the situation after 100 ps. Following its extended annealing, structure $\mathrm{E}^{\prime}$ had a $\chi$ of 0.8 and an $\mathrm{sp}^{2}$ fraction of $94.7 \%$, indicating a highly ordered structure with extended graphene sheets as seen in Fig. 2 . 
Fig. 3 shows calculations of $u$ during compression and decompression processes. A high density structure (approximately $3 \mathrm{~g} / \mathrm{cm}^{3}$ ), labeled $I$ in Fig. 3, behaves as a normal elastic material in the sense that $u$ increases as the density increases and when the pressure is released, it recovers its initial density almost completely. Examination of the bonding in case $I$ before and after the full compression and decompression cycle shows that the compression process generates a structure which is statistically similar to the original structure (in the sense that the structures have the same average coordination and $\chi$ ) even though some bonds have been broken (10.4\% out of the initial 3533 bonds are broken) and new bonds have formed (11.8\% of the final bonds are new). In contrast, structures with low densities are unstable with respect to compression and exhibit little recovery upon decompression (see $B$ and $E$ in Fig. 3). A picture of the structure at B (density 2 $\mathrm{g} / \mathrm{cm}^{3}$ ) is shown in Fig. 2(a). Annealing of structure B, however, converts it to a normal elastic material that shows recovery upon decompression (see B' in Fig. 3). Inspection of structure B' before and after deformation shows that the topology of its $s p^{2}$ bonded sheets has been preserved almost exactly (only $0.5 \%$ of the original bonds are broken, and in the final structure just $0.9 \%$ of the bonds are new). The memory of the initial structure is retained by the topology-preserving properties of graphene sheets (which are clearly visible in Fig. 2(b)) [31]. 


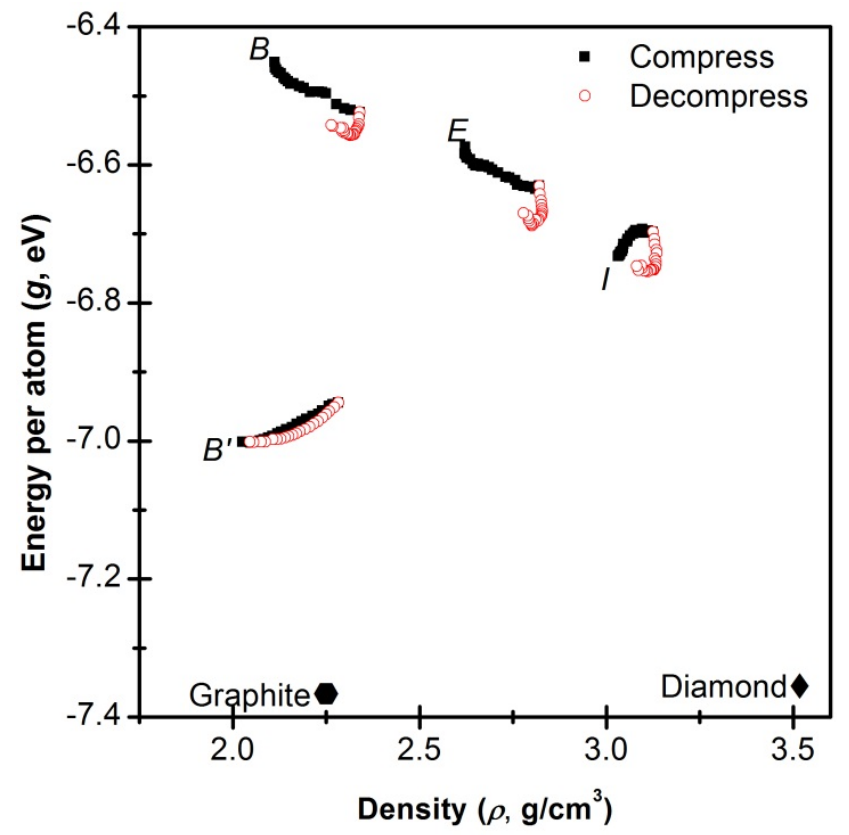

Fig. 3 - The progression of the free energy per atom $(g)$ and density $(\rho)$ during compression and decompression processes for four starting structures $B, E, I$ and $B$ ' shown in Fig. 1.

Fig. 4(a) shows a proposed schematic three-dimensional landscape of the energy of carbon solids at $\mathrm{P}=0$ and $\mathrm{T}=0$, expressed as a function of $\rho$ and $\chi$. Although the energy values have not been directly computed from structures and are expressed in arbitrary units, the shape of the landscape has been drawn to preserve the relative energies of the structures for which values of $u$ have been computed. The shape of the landscape at the back plane $(\chi=0)$ is described by the potential energies of the as-quenched structures (Fig. 1(b)) which have the highest degree of structural disorder. The front plane $(\chi=1)$ of the landscape displays the potential energies of fully crystalline carbon solids. The landscape has two valleys, one representing the $\mathrm{sp}^{2}$ bonded forms that include graphite and its polytypes and the other representing the $\mathrm{sp}^{3}$ bonded forms of diamond and its polytypes. The crystalline structures at densities other than those of graphite and diamond contain strained bonds and are shown as having much higher energy than graphite and diamond. The spacing between time points in Fig. 1 is a measure of the driving force for energy reduction and was used as a guide in determining the slope of the landscape in Fig. 4(a). 

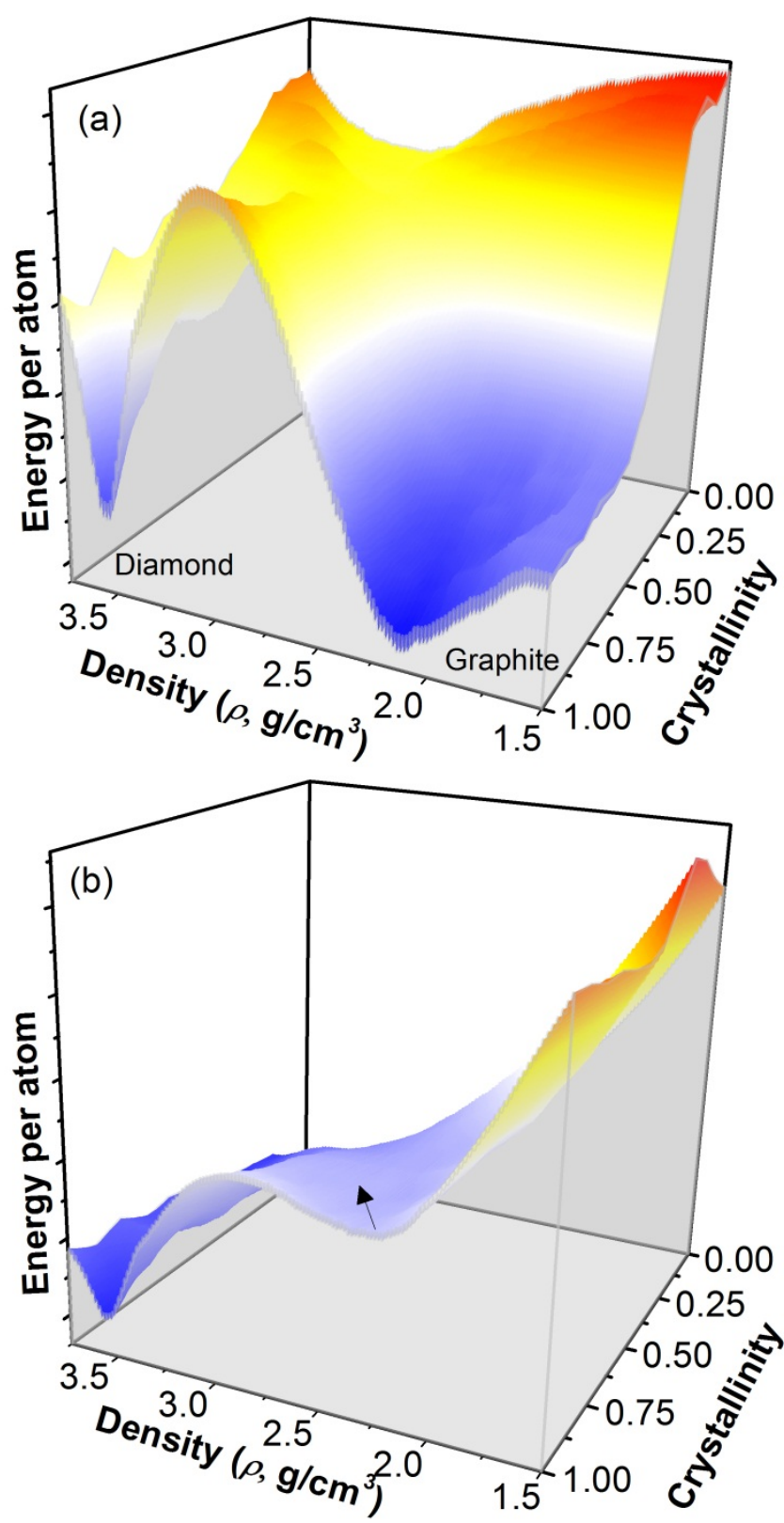

Fig. 4 - (a) Schematic representation of the energy landscape for carbon at zero $P$ and $T$, based on the simulations of Figs. 1 and 2. The locations of crystalline diamond and graphite are indicated. (b) The landscape of (a) at non-zero $P$ and $T$ calculated from (a) using equation (1). This is tilted to favour high density and high entropy (disordered) structures. The arrow in (b) shows the transformation from a well ordered graphitic carbon to a high-density $a-C$ under the application of high pressures and moderate temperatures. 
The shape of the landscape is useful in describing observations of annealing experiments. Where the slope of the landscape is small, downward progression during annealing is expected to slow down and may stop in a local minimum from which it cannot escape except by increasing the annealing temperature. Escape from local minima is difficult if the available thermal energy at the annealing temperature is less than the energy differences between neighboring structures in the landscape. These energy differences are illustrated schematically as a roughness of the landscape surface in Fig. 4. The landscape of Fig. 4(a) is intended to represent the structure of minimum potential energy for a given choice of structural parameters $\rho$ and $\chi$. There will be other possible structures with a higher energy that have the same values of $\rho$ and $\chi$ as the minimum energy structure.

Experimental observations directly support predictions derived from the landscape. When interpreting changes induced by the application of pressure and temperature in terms of the landscape, the following procedure is used. Pressure and temperature are changed by a process and the structure evolves on a modified landscape, appropriate to the $\mathrm{P}$ and $\mathrm{T}$ that apply during the process. The modified landscape can be calculated from the zero $\mathrm{P}$ and $\mathrm{T}$ landscape using equation (1). Increasing the pressure in equation (1) results in a tilting of the landscape in which lower density structures are preferentially raised to higher energies. Increasing the temperature lowers the energy of amorphous structures with high entropy s, (ie high structural disorder), relative to crystalline structures with low structural disorder. The parameter $\chi$ is a measure of structural order and is therefore inversely related to $s$. An example of a landscape applying at elevated pressures and moderate temperatures is shown in Fig. 4(b).

It has been observed that compression in diamond anvils at room temperature of crystalline and noncrystalline graphitic carbons leads to reversible transitions to higher density structures which have been found to be amorphous [13] and transparent [9]. Because of the high energy ridge between the graphite and diamond valleys shown in Fig. 4(b), an ordered graphitic structure will move towards the back plane 
(amorphize) rather than transform into diamond (this pathway is shown as an arrow in Fig. 4(b)). Note that once pressure is removed, the landscape returns to its original form (Fig. 4(a)) so that the high density amorphous structure has the possibility of reverting to its low density graphitic form, since a low temperature pathway exists from the original to the final structures. The energy barriers along the pathway are small since it occurs at room temperature, making a reversible process likely. The landscape shows that the high density amorphous transparent forms produced at high pressures are located close to the position of $t a-\mathrm{C}$ and are therefore likely to be structurally similar to $t a-\mathrm{C}$.

If a process involves application of pressure at elevated temperature, significant energy barriers may be overcome during the process so that the pathway for the transformation is not necessarily reversible at room temperature, even if the final state is at a higher energy location on the zero $\mathrm{P}, \mathrm{T}$ landscape. The recent observation [32] that shock compression of neutron irradiated (disordered) highly oriented pyrolytic graphite (HOPG) transforms irreversibly into amorphous diamond (a term equivalent to ta-C) whereas as-received HOPG only suffers reversible transformation is a natural prediction of our landscape approach. High P high T conditions tilt the landscape to the left as in Fig. 4(b) but with a further tilt towards the rear, more strongly favoring highly $\mathrm{sp}^{3}$ amorphous forms. Disordering the HOPG places the starting point for the transformation closer to the highly $\mathrm{sp}^{3}$ amorphous forms, allowing it to transform more completely into the $\mathrm{sp}^{3}$ disordered structures during the rapid shock process. After the compression is removed, the pathway for the disordered HOPG will not readily reverse in the available time, whereas for ordered HOPG the shorter return path and steeper slope of the landscape encourage reversal.

The topology of the landscape of Fig. 4(a) is shown in Fig. 5 as a contour plot in the $\rho-\chi$ plane. The contour plot is useful for explaining the results of indenting non crystalline carbon at room temperature with sharp tips. Compression is equivalent to a movement mainly across the landscape parallel to the $\rho$ axis, assuming the changes in bonding do not give rise to significant changes in $\chi$. The compression trajectory indicated by 
the arrow labeled $\alpha$ in Fig. 5 reduces the free energy and the compression is therefore not reversible. An example of this behavior is the compression of structures $B$ and $E$ in Fig. 3. Compression of a structure at a higher density (labeled $\beta$ in Fig. 5) shows an increase in energy following the compression and since the landscape slope is parallel to the arrow $\beta$, the structure returns approximately to its initial location when the compression is released (an example of this behavior is seen in case $I$ in Fig. 2). Compression of an ordered low-density structure (labeled $\gamma$ in Fig. 4) also shows an increase in energy after compression, a behavior indicative of an elastic medium, an example of which is case $B^{\prime}$ shown in Fig. 2. Note that the indentation of highly crystalline materials may not be well explained by a Gibbs free energy in which the forces are purely hydrostatic as in (1). The observation [33] that indentation-induced shear forces produce a transformation of diamond to graphite therefore would not necessarily be deduced from the landscape of Fig. 3 . The landscape predicts that compression of diamond at low temperature is reversible, but diamond could be amorphized to ta-C at high pressures and temperatures.

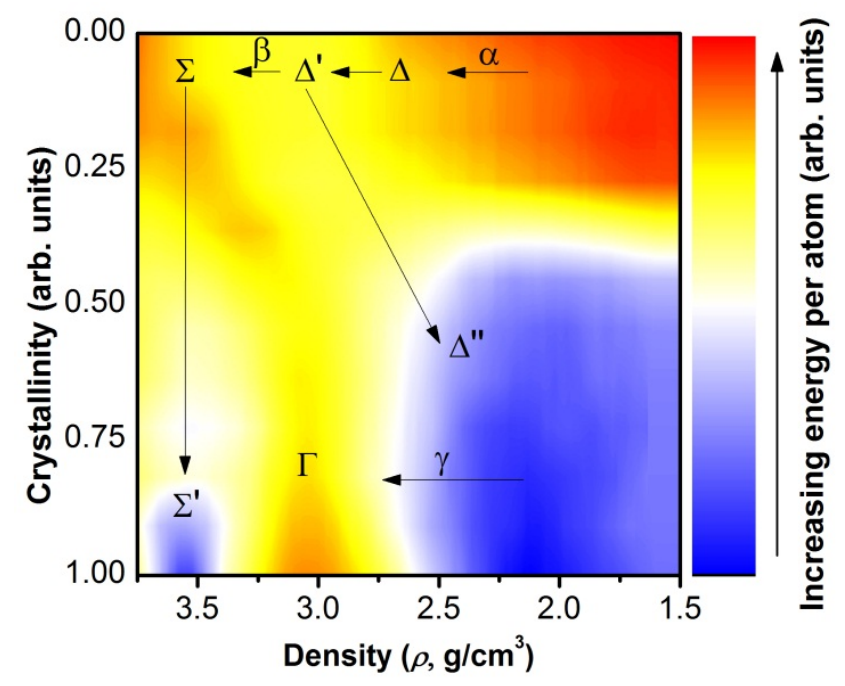

Fig. 5 - Contour plot of the energy landscape shown in Fig. 3(a) in the density-crystallinity plane. As described in the text, the symbols $\Gamma, \Delta, \Sigma$ refer to locations while $\alpha, \beta$ and $\gamma$ refer to trajectories. 
The landscape also explains observations in carbon thin film synthesis. An amorphous film with a density of $2.7 \mathrm{~g} / \mathrm{cm}^{3}$, typically grown from a carbon plasma at room temperature [18], corresponds to point $\Delta$ in Fig. 5. Growth under the same deposition conditions on a substrate heated to $150^{\circ} \mathrm{C}$ has been observed to result in a reduction in stress and a carbon film of higher density than observed for growth at room temperature. This situation is shown in Fig. 4 as the arrow linking $\Delta$ and $\Delta^{\prime}$. The use of the zero $\mathrm{P}$ and $\mathrm{T}$ landscape is appropriate once the stress has been relieved. Since $\Delta \rightarrow \Delta^{\prime}$ gives a decrease in free energy, this process is expected to occur during dynamic annealing. A similar effect in carbon films has been observed using post deposition annealing [19]. Growth under the same conditions but at higher temperatures of $500-600^{\circ} \mathrm{C}$ allows dynamic annealing to proceed further, resulting a more extensive evolution of structure into the graphite valley (trajectory $\Delta \rightarrow \Delta^{\prime} \rightarrow \Delta^{\prime \prime}$ ). Since the initial point on this trajectory has a higher density than the final point, an expansion of the structure will occur that results in an increase in the compressive stress that has been observed experimentally [18]. Point $\Gamma$ in Fig. 5 corresponds to a partially ordered structure with intermediate density lying on the ridge between the graphite and diamond valleys. We predict that such structures will not be observed in film growth experiments since there is no downhill route to them from any possible structure as deposited from vapor or plasma.

An interesting question to contemplate is whether there are any growth conditions that will allow a structure prepared from carbon vapor to progress directly down the diamond valley, leading to well-formed diamond crystals of macroscopic dimensions. Our zero P and T landscape, shows diamond formation from vapor will not occur at low pressures since there is no downward pathway into the diamond valley from the back (zero crystallinity) axis. However, a pressure- and temperature- tilted landscape of the type shown in Fig. 4(b) would could possibly lead to the trajectory $\Sigma \rightarrow \Sigma$ ' which leads downwards in the diamond valley as shown in Fig. 5. The conversion of carbon onions to crystalline diamond has been shown to occur under electron irradiation which is believed to create a defective $\mathrm{sp}^{2}$ bonded structure that transforms to diamond under the pressure applied by the contacting graphitic shells [34]. Our landscape shows that the core of the onion could 
transform first to an amorphous $\mathrm{sp}^{3}$ rich phase that with annealing under pressure is able to move down the diamond valley to form well crystallized diamond.

\section{Conclusion}

We have constructed an energy landscape for carbon network solids expressed as function of density and crystallinity, based on the results of atomistic simulations. The landscape has been used to explain many observations of the changes in carbon structures that follow mechanical deformation, thermal annealing and shock compression. The landscape explains why some carbon structures fully recover from deformation and why room temperature compression of graphitic carbon can lead to reversible amorphization. The landscape also explains how annealing can both increase and decrease the density of carbon films and why it is not possible to form diamond at low pressures from carbon vapor alone.

\section{Acknowledgments}

The authors gratefully acknowledge support provided by the Australian Research Council (ARC). 


\section{References}

[1] Harris PJF. New Perspectives on the Structure of Graphitic Carbons. Critical Reviews in Solid State and Materials Sciences. 2005;30(4):235-53.

[2] Powles RC, Marks NA, Lau DWM. Self-assembly of $\mathrm{sp}^{\wedge}\{2\}$-bonded carbon nanostructures from amorphous precursors. Phys Rev B. 2009;79(7):075430.

[3] Townsend SJ, Lenosky TJ, Muller DA, Nichols CS, Elser V. Negatively curved graphitic sheet model of amorphous carbon. Phys Rev Lett. 1992;69(6):921-4.

[4] Jenkins GM, Jouquet G. The effect of short-term neutron irradiation on the shear compliance of hotworked pyrolytic graphite. Carbon. 1968;6(1):85-91.

[5] Jenkins GM, Kawamura K. Polymeric Carbons-Carbon Fibre Glass and Char. London: Cambridge University Press; 1976.

[6] Robertson J. Amorphous carbon. Advances in Physics. 1986;35(4):317-74.

[7] McKenzie DR, Muller D, Pailthorpe BA. Compressive-stress-induced formation of thin-film tetrahedral amorphous carbon. Phys Rev Lett. 1991;67(6):773-6.

[8] Bundy FP, Bassett WA, Weathers MS, Hemley RJ, Mao HU, Goncharov AF. The pressuretemperature phase and transformation diagram for carbon; updated through 1994. Carbon. 1996;34(2):14153.

[9] Badding JV, Lueking AD. Reversible high pressure sp2-sp3 transformations in carbon. Phase Transitions. 2007;80(10-12):1033-8.

[10] Hanfland M, Beister H, Syassen K. Graphite under pressure: Equation of state and first-order Raman modes. Phys Rev B. 1989;39(17):12598-603.

[11] Mao WL, Mao H-k, Eng PJ, Trainor TP, Newville M, Kao C-c, et al. Bonding Changes in Compressed Superhard Graphite. Science. 2003;302(5644):425-7.

[12] Goncharov AF. Graphite at high pressures: Amorphization at $44 \mathrm{GPa}$. High Pressure Research. 1992;8(4):607-16. 
[13] Lin Y, Zhang L, Mao H-k, Chow P, Xiao Y, Baldini M, et al. Amorphous Diamond: A High-Pressure Superhard Carbon Allotrope. Phys Rev Lett. 2011;107(17):175504.

[14] Martin PJ, Filipczuk SW, Netterfield RP, Field JS, Whitnall DF, McKenzie DR. Structure and hardness of diamond-like carbon films prepared by arc evaporation. J Mater Sci Lett. 1988;7(4):410-2.

[15] Iwashita N, Swain MV, Field JS, Ohta N, Bitoh S. Elasto-plastic deformation of glass-like carbons heat-treated at different temperatures. Carbon. 2001;39(10):1525-32.

[16] Ferrari AC, Kleinsorge B, Morrison NA, Hart A, Stolojan V, Robertson J. Stress reduction and bond stability during thermal annealing of tetrahedral amorphous carbon. J Appl Phys. 1999;85(10):7191-7.

[17] Chhowalla M, Robertson J, Chen CW, Silva SRP, Davis CA, Amaratunga GAJ, et al. Influence of ion energy and substrate temperature on the optical and electronic properties of tetrahedral amorphous carbon (ta-C) films. J Appl Phys. 1997;81(1):139-45.

[18] Lau DWM, Moafi A, Taylor MB, Partridge JG, McCulloch DG, Powles RC, et al. The structural phases of non-crystalline carbon prepared by physical vapour deposition. Carbon. 2009;47(14):3263-70.

[19] McCulloch DG, Peng JL, McKenzie DR, Lau SP, Sheeja D, Tay BK. Mechanisms for the behavior of carbon films during annealing. Phys Rev B. 2004;70(8):085406.

[20] Gaudin J, Peyrusse O, Chalupský J, Toufarová M, Vyšín L, Hájková V, et al. Amorphous to crystalline phase transition in carbon induced by intense femtosecond x-ray free-electron laser pulses. Phys Rev B. 2012;86(2):024103.

[21] McKenzie DR. Tetrahedral bonding in amorphous carbon. Reports on Progress in Physics. 1996;59(12):1611.

[22] Raoux S, Wełnic W, Ielmini D. Phase Change Materials and Their Application to Nonvolatile Memories. Chem Rev. 2009;110(1):240-67.

[23] Marks NA. Generalizing the environment-dependent interaction potential for carbon. Phys Rev B. 2000;63(3):035401. 
functional, tight-binding, and empirical methods for the simulation of amorphous carbon. Phys Rev B. 2002;65(7):075411.

[25] Marks NA. Thin film deposition of tetrahedral amorphous carbon: a molecular dynamics study. Diamond Relat Mater. 2005;14(8):1223-31.

[26] Lau DWM, McCulloch DG, Marks NA, Madsen NR, Rode AV. High-temperature formation of concentric fullerene-like structures within foam-like carbon: Experiment and molecular dynamics simulation. Phys Rev B. 2007;75(23):233408.

[27] Marks NA, Lattemann M, McKenzie DR. Nonequilibrium Route to Nanodiamond with Astrophysical Implications. Phys Rev Lett. 2012;108(7):075503.

[28] Suarez-Martinez I, Marks NA. Amorphous carbon nanorods as a precursor for carbon nanotubes. Carbon. 2012;50(15):5441-9.

[29] Suarez-Martinez I, Higginbottom PJ, Marks NA. Molecular dynamics simulations of the transformation of carbon peapods into double-walled carbon nanotubes. Carbon. 2010;48(12):3592-8.

[30] Colonna F, Los JH, Fasolino A, Meijer EJ. Properties of graphite at melting from multilayer thermodynamic integration. Phys Rev B. 2009;80(13):134103.

[31] Iwashita N, Field JS, Swain MV. Indentation hysteresis of glassy carbon materials. Phil Mag A. 2002;82(10):1873-81.

[32] Niwase K, Nakamura KG, Yokoo M, Kondo K-i, Iwata T. Pathway for the Transformation from Highly Oriented Pyrolytic Graphite into Amorphous Diamond. Phys Rev Lett. 2009;102(11):116803.

[33] Gogotsi YG, Kailer A, Nickel KG. Materials: Transformation of diamond to graphite. Nature. 1999;401(6754):663-4.

[34] Banhart F. The transformation of graphitic onions to diamond under electron irradiation. J Appl Phys. 1997;81(8):3440-5. 